\title{
RECENT RESULTS OF THE CPLEAR EXPERIMENT
}

\section{The CPLEAR Collaboration}

R. Adler ${ }^{2}$, T. Alhalel ${ }^{2}$, A. Angelopoulos ${ }^{1}$, A. Apostolakis ${ }^{1}$, E. Aslanides ${ }^{11}$, G. Backenstoss ${ }^{2}$, C.P. Bee ${ }^{11}$, O. Behnke ${ }^{17}$, J. Bennet ${ }^{9}$, V. Bertin ${ }^{11}$, F. Blanc ${ }^{7,13}$, P. Bloch ${ }^{4}$, Ch. Bula ${ }^{13}$, P. Carlson ${ }^{15}$, M. Carroll ${ }^{9}$

J. Carvalho ${ }^{5}$, E. Cawley ${ }^{9}$, S. Charalambous ${ }^{16}$, M. Chardalas ${ }^{16}$, G. Chardin ${ }^{14}$, M.B. Chertok ${ }^{3}$, M. Danielsson ${ }^{15}$, A. Cody $^{9}$, S. Dedoussis ${ }^{16}$, M. Dejardin ${ }^{4}$, J. Derre ${ }^{14}$, M. Dodgson ${ }^{9}$, J. Duclos ${ }^{14}$, A. Ealet ${ }^{11}$, B. Eckart ${ }^{2}$, C. Eleftheriadis ${ }^{16}$, I. Evangelou ${ }^{8}$, L. Faravel ${ }^{7,11}$, P. Fassnacht ${ }^{11}$, J.L. Faure ${ }^{14}$, C. Felder ${ }^{2}$,

R. Ferreira-Marques ${ }^{5}$, W. Fetscher ${ }^{17}$, M. Fidecaro ${ }^{4}$, A. Filipčic ${ }^{10}$, D. Francis ${ }^{3}$, J. Fry', E. Gabathuler ${ }^{9}$, R. Gamet ${ }^{9}$, D. Garreta ${ }^{14}$, T. Geralis ${ }^{13}$, H.-J. Gerber ${ }^{17}$, A. Go $^{3}$, P. Gumplinger $^{17}$, C. Guyot ${ }^{14}$, A. Haselden ${ }^{9}$, P.J. Hayman ${ }^{9}$, F. Henry-Couannier ${ }^{11}$, R.W. Hollander ${ }^{6}$, E. Hubert ${ }^{11}$, K. Jansson ${ }^{15}$, H.U. Johner ${ }^{7}$, K. Jon-And ${ }^{15}$, P.R. Kettle ${ }^{13}$, C. Kochowski ${ }^{14}$, P. Kokkas ${ }^{8}$, R. Kreuger ${ }^{6}$, T. Lawry ${ }^{3}$, R. Le Gac ${ }^{11}$, F. Leimgruber ${ }^{2}$, A. Liolios ${ }^{16}$, E. Machado ${ }^{5}$, P. Maley ${ }^{9}$, I. Mandić ${ }^{10}$, N. Manthos ${ }^{8}$, G. Marel ${ }^{14}$, M. Mikuž $^{10}$, J. Miller ${ }^{3}$, F. Montanet ${ }^{11}$, T. Nakada ${ }^{13}$, A. Onofre ${ }^{5}$, B. Pagels ${ }^{17}$, P. Pavlopoulos ${ }^{2}$, F. Pelucchi ${ }^{11}$, J. Pinto da Cunha $^{5}$, A. Policarpo ${ }^{5}$, G. Polivka ${ }^{2}$, H. Postma ${ }^{6}$, R. Rickenbach ${ }^{2}$, B.L. Roberts ${ }^{3}$, E. Rozaki ${ }^{1}$, T. Ruf ${ }^{4}$, L. Sacks ${ }^{9}$, L. Sakeliou ${ }^{1}$,P. Sanders', C. Santoni ${ }^{2}$, K. Sarigiannis ${ }^{1}$, M. Schäfer ${ }^{17}$, L.A. Schaller ${ }^{7}$, A. Schopper ${ }^{4}$, P. Schune ${ }^{14}$, A. Soares ${ }^{14}$, L. Tauscher ${ }^{2}$, C. Thibault ${ }^{12}$, F. Touchard ${ }^{4}$, C. Touramanis ${ }^{9}$, F. Triantis ${ }^{8}$, D.A. Tröster ${ }^{2}$, E. Van Beveren ${ }^{5}$, C.W.E. Van Eijk ${ }^{6}$, S. Vlachos ${ }^{2}$, P. Weber ${ }^{17}$, O. Wigger $^{13}$, C. Witzig ${ }^{17}$, M. Wolter ${ }^{17}$, C. Yeche ${ }^{14}$, D. Zavrlanik ${ }^{10}$ and D. Zimmerman ${ }^{3}$.

${ }^{1}$ University of Athens, ${ }^{2}$ University of Basle, ${ }^{3}$ Boston University, ${ }^{4} \mathrm{CERN},{ }^{5} \mathrm{LIP}$ and University of Coimbra, ${ }^{6}$ Delft University of Technology, ${ }^{7}$ University of Fribourg, ${ }^{8}$ University of Ioannina, ${ }^{9}$ University of Liverpool,

${ }^{10} \mathrm{~J}$. Stef an Inst. and Phys. Dep., University of Ljubljana, ${ }^{11}$ CPPM-IN2P3-CNRS et Université

d'Aix-Marseille II, ${ }^{12}$ CSNSM-IN2P3-CNRS, ${ }^{13}$ Paul-Scherrer-Institut(PSI), ${ }^{14}$ DAPNIA/SPP CE Saclay,

${ }^{15} \mathrm{KTH}$ Stockholm, ${ }^{10}$ University of Thessaloniki, ${ }^{17}$ ETH-ITP Zürich

Presented by R. LE GAC

IN2P3-CPPM, 163 av. de Luminy, case 905, F-13288 MARSEILLE CEDEX 9

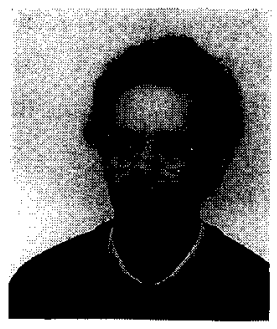

Abstract

The CPLEAR experiment running at CERN, measures CP T and CPT,violating parameters in the neutral kaon system by measuring time dependent decay asymmetries between $\mathrm{K}^{0}$ and $\overline{\mathrm{K}^{0}}$. Physics results corresponding to about $25 \%$ of the presently available statistics are presented for the $\pi^{+} \pi^{-}$ and the $\pi^{+} \pi^{-} \pi^{0}$ decay channels. The present precisions are already at the level of the world average value for $\phi_{+-}$and one order of magnitude better than the published results for $\eta_{+-0}$. Moreover, for the first time the amplitude for the CP allowed $\mathrm{K}_{S}$ decay into $\pi^{+} \pi^{-} \pi^{0}$ has been evaluated. 


\section{6}

\section{INTRODUCTION}

The CPLEAR experiment aims to measure the $\mathrm{CP}, \mathrm{T}$ violation effects through the observation of particle-antiparticle decay rate asymmetries by detecting initial $\mathrm{K}^{0}$ and $\overline{\mathrm{K}^{0}}$ states and their decay products: $\tau^{+} \pi^{-}, \pi^{+} \pi^{-} \pi^{0}$ and $\pi \nu l$.

The decay rate of initial $\mathrm{K}^{0}\left(\overline{\mathrm{K}^{0}}\right)$ into $\pi^{+} \pi^{-*}$ assuming $\epsilon_{S}=\epsilon_{L}=\epsilon$, is given by (see the previous presentation for the definition of the parameters):

$$
R_{\mathrm{K}^{0}} \frac{\mathrm{K}^{0}(t)}{(t)} \propto[1 \mp \operatorname{Re}(\epsilon)]\left[e^{-\gamma_{s} t}+\left|\eta_{+-}\right|^{2} e^{-\gamma_{L} t} \pm 2\left|\eta_{+-}\right| e^{\frac{-\left(\gamma_{s}+\gamma_{L}\right) t}{2}} \cos \left(\Delta \mathrm{m} t-\phi_{+-}\right)\right]
$$

The mass difference $\Delta \mathrm{m}$ between $\mathrm{K}_{S}$ and $\mathrm{K}_{L}$ is approximately equal to half of the decay width of the $\mathrm{K}_{S}$ and the interference amplitude is observable between 5 and $15 \tau_{s}$. CPLEAR measures the time dependent asymmetry between $\overline{\mathrm{K}^{0}}$ and $\mathrm{K}^{0}$ :

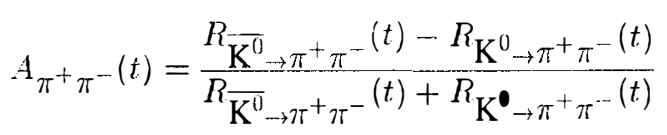

which is sensitive to $\Delta \mathrm{m}$ and the $\mathrm{CP}$ violation parameters $\left|\eta_{+--}\right|$and $\phi_{+--}$:

$$
A_{\pi^{+} \pi^{-}}(t) \approx 2 \operatorname{Re}(\epsilon)-\frac{2\left|\eta_{+--}\right| e^{\frac{\left(\eta_{S}-\gamma_{L}\right) t}{2}} \cos \left(\Delta \mathrm{m} t-\phi_{+-.}\right)}{1+\left|\eta_{+--}\right|^{2} e^{\left(\gamma_{i}-\gamma_{L}\right) t}}
$$

The detector efficiencies, geometrical acceptance and residual background are identical for $\mathrm{K}^{0}$ and $\overline{\mathrm{K}^{0}}$ decay and therefore cancel in the asymmetry. This measurement allows to evaluate both the amplitude and the phase of the $\mathrm{CP}$ violation parameter in the $\pi^{+} \pi^{-}$decay channel. Moreover, the conservation of CP $\Gamma$ can be tested by comparing $\phi_{+-}$with the superweak angle $\phi_{S W}$ defined as $\arctan \left(2 \Delta \mathrm{m} /\left(\gamma_{S}-\gamma_{L}\right)\right)$. If CPT is a good symmetry $\phi_{+--} \approx \phi_{\mathrm{SW}}$. The present value for ${ }_{\mathrm{SW}}$ is $(43.7 \pm 0.2)^{\circ}[1]$.

Time dependent asymmetries probe the $\mathrm{CP}$ violation even if the final state is not a pure CP eigenstate and allow to measure $\eta_{+-0}$ in the $\pi^{+} \pi^{-\cdots} \pi^{0}$ decay.

\section{THE CPLEAR EXPERIMENT}

The CPLEAR detector, shown on Figure 1, allows to tag the strangeness of the neutral kaon and to measure its decay product. It has a cylindrical geometry and is mounted inside a solenoid of $3.6 \mathrm{~m}$ length and $1 \mathrm{~m}$ radius, which produces a magnetic field of $0.44 \mathrm{~T}$ parallel to the antiproton beam. Antiprotons coming from LEAR with a momentum of $200 \mathrm{MeV} / \mathrm{c}$ annihilate at rest, at the center of the detector, in a spherical target filled with hydrogen at a pressure of 16 bars. $\mathrm{K}^{0}$ and $\overline{\mathrm{K}^{0}}$ are produced in $\approx 410^{-3}$ of the annihilations through the reactions:

$$
\mathrm{p} \overline{\mathrm{p}} \rightarrow \mathrm{K}^{+} \pi^{-} \overline{\mathrm{K}^{0}} \text { or } \mathrm{K}^{--} \pi^{+} \mathrm{K}^{0}
$$




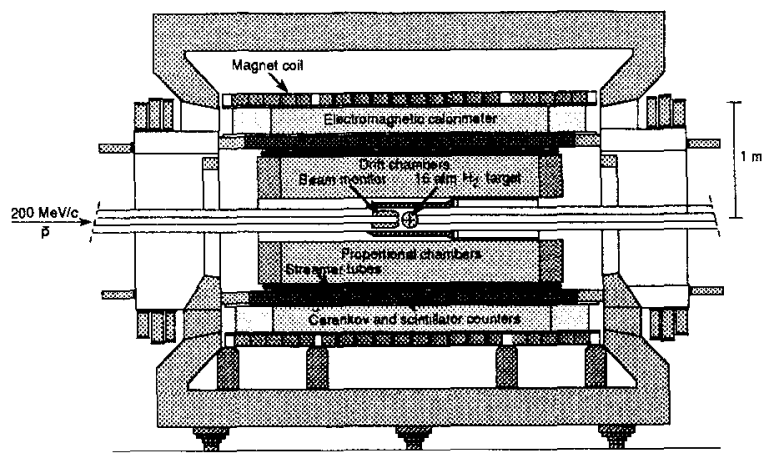

Figure 1: CPLEAR detector

In these reactions, the identification of the charged kaon and of its charge sign determines the strangeness of the associated neutral kaon.

The target is surrounded by a tracking device consisting of 2 proportional chambers, 6 drift chambers and 2 layers of streamer tubes. This detector allows the reconstruction of the charged particle tracks and the measurement of their momentum. It is followed by a particle identification device, made of a threshold Cherenkov sandwiched between 2 layers of scintillators, which separates charged kaons (which do not produce light in the Cherenkov) from charged pions. Finally an 18-layer gas-sampling calorimeter is used to detect gammas. With its high granularity, the impact points of the showers are reconstructed accurately.

To face the high rate $(1 \mathrm{MHz})$ required to measure accurately the $\mathrm{CP}$ violation parameters and to select the reaction (2) among the much more numerous multipionic annihilations, a sophisticated multi-level trigger is required. It is based on the recognition of the associated charged $\mathrm{K} \pi$ pair at the annihilation vertex. The first requirement of the trigger is that there are at least two hits in the inner scintillator layer and at least one charged kaon candidate in the Cherenkov. The next trigger stages place kinematical constraints on the event firstly by applying a cut to the transverse momentum of the kaon candidate, and then by requiring 2 or 4 tracks after a full event reconstruction and track parameterization. The following processor improves the particle identification using the energy loss of the particles in the scintillators, the time of flight difference between kaons and pions and the number of photo-electrons in the Cherenkov. The last trigger stage requires a minimum number of clusters in the calorimeter for two-tracks events. The maximum trigger decision time is around $34 \mu \mathrm{s}$ and its reduction factor is about 1000 . 


\section{8}

\section{$2 \quad \mathbf{K}^{0}$ DECAY INTO $\pi^{+} \pi^{-}$}

The identification of the $\mathrm{K}^{0}$ decay into $\pi^{+} \pi^{-}$is based on constrained fits. The constraints are the following:

- the missing mass to the $\mathrm{K}^{ \pm} \pi^{\mp}$ pair at the annihilation vertex should be equal to the $\mathrm{K}^{0}$ mass.

- the total energy of the 4 particles should be equal to twice the proton mass and the total momentum should be equal to zero.

- the $\mathrm{K}^{0}$ flight direction should agree with the direction between the annihilation and the decay vertex.

The present analysis used data recorded up to the end of 1992. After the fits, $210^{6}$ events are selected with a decay time above $2 \tau_{s}$. In the final sample the remaining background is very small. It is only present at large decay time and comes mainly from the $\mathrm{K}_{L}$ decay into $\pi \nu l$. It has been evaluated to be about $1.21 \pm 0.14$ larger than the $\mathrm{CP}$ violating $\mathrm{K}_{L}$ decay into $\pi^{+} \pi^{-}$.

Even if $\mathrm{K}^{0}$ and $\overline{\mathrm{K}^{0}}$ are produced symmetrically, the number of reconstructed $\mathrm{K}^{0}$ and $\overline{\mathrm{K}^{0}}$ is not equal because the detector efficiencies of $\mathrm{K}^{+}$and $\mathrm{K}^{-}$differ in the detector due to their different nuclear cross-section. This effect modifies the relative proportion of $\mathrm{K}^{0}$ and $\overline{\mathrm{K}^{0}}$ in our sample but we have checked that this proportion does depend neither on the $\mathrm{K}^{0}$ momentum nor on the $\mathrm{K}^{0}$ decay time. It is taken into account by introducing a normalization factor $1.1187 \pm 0.0026$ measured on $\mathrm{K}^{0} \rightarrow$ $\pi^{+} \pi^{-}$events with a decay time between 2 and $4 \tau_{s}$ where the CP violating effects are very small.

The reconstructed time dependent asymmetry is shown on Figure 2a together with the results of the fit. The fit of the asymmetry to expression (1), using a fixed value of $\gamma_{S}, \gamma_{L}$ and $\Delta \mathrm{m}\left(0.5351 \pm 0.002410^{10} \overline{\mathrm{h}} / \mathrm{s}\right)$ from reference [1] and taking into account the remaining background, gives the following values for the $\mathrm{CP}$ violation parameters:

$$
\left\{\begin{array}{l}
\eta_{+-}=\left(2.25 \pm 0.0 \bar{\tau}_{\text {stat }} \pm 0.02_{\text {sys }}\right) 10^{-3} \\
\phi_{+-}=\left(44 . \bar{\tau} \pm 1.3_{\text {stat }} \pm 0 . i_{\text {sys }} \pm 0 . \tau_{\Delta \mathrm{m}}\right)^{o}
\end{array}\right.
$$

The quoted systematic errors are preliminary. They take into account the uncertainties linked to the correction for the regeneration effects and to the $\mathrm{K}^{0}, \overline{\mathrm{K}^{0}}$ normalization. The error related to the uncertainty on $\Delta \mathrm{m}$ will be reduced in the future due to the measurement of $\Delta \mathrm{m}$ which will be obtained in our experiment.

\section{$3 \quad \mathbf{K}^{0}$ DECAY INTO $\pi^{+} \pi^{-} \pi^{0}$}

In this analysis, events are selected by using the following criteria: 

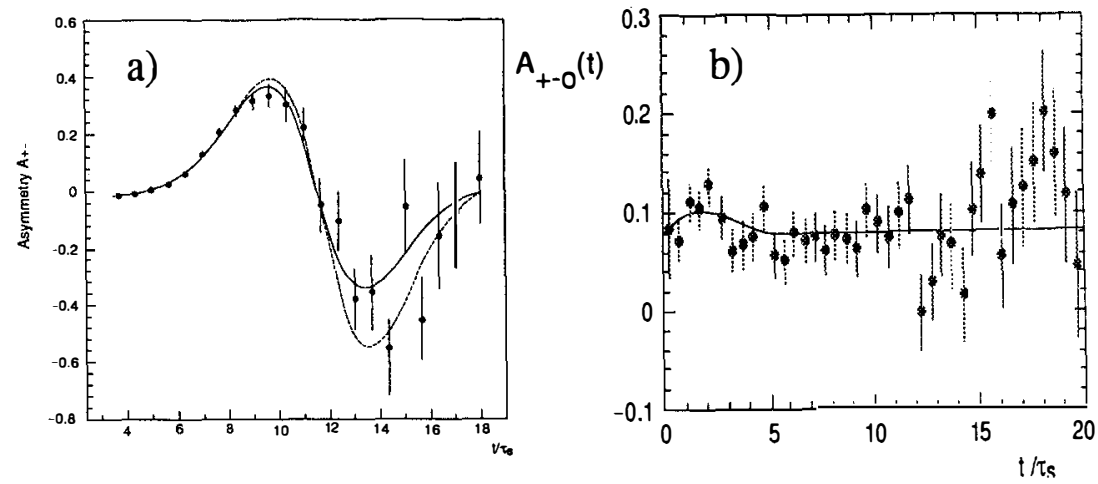

Figure 2: a) Time dependent asymmctry for $\mathrm{K}^{0}$ decay into $\pi^{+} \pi^{-}$. The solid (dotted) line is the result of a fit with (without) taking into account the residual three-body background. b) Time dependent asymmetry for $\mathrm{K}^{0}$ decay into $\pi^{+} \pi^{-} \pi^{0}$.

- the missing mass to the $\mathrm{K}^{ \pm} \pi^{\mp}$ pair should be equal to the $\mathrm{K}^{\mathbf{0}}$ mass.

- the missing mass to the 4 detected charged particles should be equal to the $\pi^{0}$ mass.

- the $\mathrm{K}^{0}$ flight direction should agree with the direction between the annihilation and the decay vertex.

- the event should not satisfy the kinematics of $\mathrm{K}^{0} \rightarrow \pi^{+} \pi^{-}$.

- at least one photon should be detected in the calorimeter.

After selection, about 36000 events remain with a background level of $15 \%$. The time dependent asymmetry $\mathrm{A}_{+-0}$ allows a measurement of $\operatorname{Re}\left(\eta_{+-0}\right)$ and $\operatorname{Im}\left(\eta_{+-0}\right)$ :

$$
A_{+-0}(t) \approx 2 \operatorname{Re}\left(\epsilon_{S}\right)-2\left(\operatorname{Re}\left(\eta_{+-0}\right) \cos (\Delta \mathrm{m} t)-\operatorname{Im}\left(\eta_{+-0}\right) \sin (\Delta \mathrm{m} t)\right) e^{\frac{-\left(\gamma_{S}-\gamma_{2}\right) t}{2}}
$$

The fit of the time dependent asymmetry shown on Figure $2 \mathrm{~b}$ gives the following results:

$$
\left\{\begin{array}{l}
\operatorname{Re}\left(\eta_{+-0}\right)=0.002 \pm 0.016_{\text {stat }} \\
\operatorname{Im}\left(\eta_{+-0}\right)=0.044 \pm 0.026_{\text {stat }}
\end{array}\right.
$$

The systematic errors, still under study, are estimated to be smaller than the present statistical error.

By studying separately the time dependent asymmetry for events with $x<0$ or $x>0$, where $x=\left(\mathrm{m}_{\pi^{+} \pi^{0}}^{2}-\mathrm{m}_{\pi^{-} \pi^{0}}^{2}\right) / \mathrm{m}_{\pi}^{2}$, the amplitude of the CP allowed $\mathrm{K}_{S}$ decay into $\pi^{+} \pi^{-} \pi^{0}$ can be measured (Figure 3 ). The fit performed simultaneously on both asymmetries leads to the following CP allowed decay width of the $\mathrm{K}_{S}$ :

$$
\frac{\Gamma\left(\mathrm{K}_{S} \rightarrow \pi^{+} \pi^{-} \pi^{0}\right)_{C: P=+1}}{\Gamma\left(\mathrm{K}_{L} \rightarrow \pi^{+} \pi^{-} \pi^{0}\right) C: P=-1}=(2.1 \pm 1.2) 10^{-3}
$$



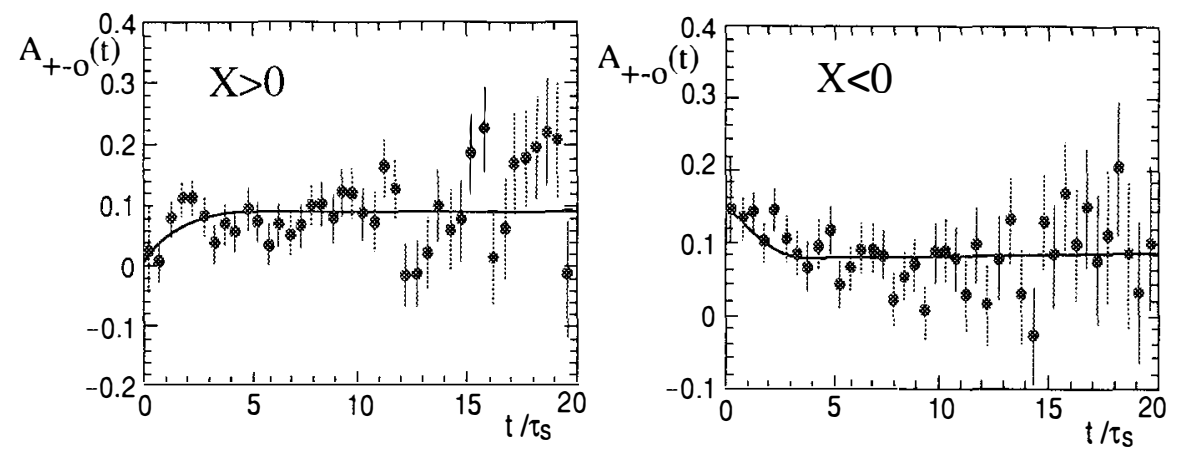

Figure 3: Time dependent asymmetry for $\mathrm{K}^{\prime \prime}$ decay into $\pi^{+} \pi^{-} \pi^{0}$ with $x>0$ and $x<0$

\begin{tabular}{|c|c|c|c|}
\hline & PDG '92 & CPLEAR '92 & CPLEAR '95 \\
\hline$\left|\eta_{+-}\right|\left[10^{-3}\right]$ & $2.279 \pm 0.022$ & $2.25 \pm 0.07$ & \pm 0.02 \\
\hline$\sigma_{+-}\left[{ }^{\circ}\right]$ & $46.5 \pm 1.2$ & $44.7 \pm 1.3$ & \pm 0.4 \\
\hline $\operatorname{Re}\left(\eta_{+-0}\right)$ & $0.05 \pm 0.17[2]$ & $0.002 \pm 0.016$ & \pm 0.004 \\
\hline $\operatorname{Im}\left(\eta_{+-0}\right)$ & $0.15 \pm 0.33[2]$ & $0.044 \pm 0.026$ & \pm 0.006 \\
\hline$\frac{\Gamma\left(\mathrm{K}_{S} \rightarrow \pi^{+} \pi^{-} \pi^{0}\right)_{C P=+1}}{\Gamma\left(\mathrm{K}_{L} \rightarrow \pi^{+} \pi^{-} \pi^{0}\right)_{C P=-1}}$ & & $(2.1 \pm 1.2) 10^{-3}$ & $\pm 0.310^{-3}$ \\
\hline
\end{tabular}

Table 1: Present and future performances of CPLEAR

\section{CONCLUSIONS}

The CPLEAR experiment measures CP violating parameters in the $\mathrm{K}^{0}$ system by measuring time dependent rate asymmetries between initial $\mathrm{K}^{0}$ and $\overline{\mathrm{K}^{0}}$. The present results are summarized in table 1 together with the world average values given in the PDG 92 [1]. The data recorded in 1993 are being analyzed and should bring an improvement of factor 4 in statistics. Running up to 1995 should allow a statistical improvement by a factor of $\approx 16$. The expected statistical precisions at the end of the experiment are listed in table 1 . The improved accuracy on $\phi_{+-}$will allow a precise test of CPT.

\section{References}

[1] Part. Data Group, Phys. Rev. D45, 1 (1992)

[2] V. V. Barmin et al., Nuovo Cimento A85, 703 (198.5) 